\title{
Robust surface states and coherence phenomena in magnetically alloyed $\mathrm{SmB}_{6}$
}

\author{
Lin Miao, ${ }^{1}$ Chul-Hee Min, ${ }^{2}$ Yishuai Xu, ${ }^{3}$ Zengle Huang, ${ }^{4}$ Erica C. Kotta, ${ }^{3}$ Rourav Basak,${ }^{3}$ \\ M. S. Song, ${ }^{5}$ B. Y. Kang, ${ }^{5}$ B. K. Cho,${ }^{5}$ K. Kißner, ${ }^{2}$ F. Reinert,${ }^{2}$ Turgut Yilmaz,${ }^{6}$ Elio

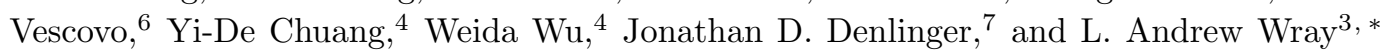 \\ ${ }^{1}$ School of Physics, Southeast University, Nanjing, 211189, China \\ ${ }^{2}$ Experimentelle Physik VII and Würzburg-Dresden Cluster of Excellence ct.qmat, \\ Universität Würzburg, Am Hubland, D-97074 Würzburg, Germany \\ ${ }^{3}$ Department of Physics, New York University, New York, New York 10003, USA \\ ${ }^{4}$ Rutgers Department of Physics and Astronomy, \\ Rutgers University, Piscataway New Jersey 08854, USA \\ ${ }^{5}$ School of Materials Science and Engineering, Gwangju Institute of Science and Technology (GIST), Gwangju 61005, Korea \\ ${ }^{6}$ National Synchrotron Light Source II, Brookhaven National Lab, Upton, New York 11973, USA \\ ${ }^{7}$ Advanced Light Source, Lawrence Berkeley National Laboratory, Berkeley, CA 94720, USA
}

(Dated: May 27, 2021)

\begin{abstract}
Samarium hexaboride is a candidate for the topological Kondo insulator state, in which Kondo coherence is predicted to give rise to an insulating gap spanned by topological surface states. Here we investigate the surface and bulk electronic properties of magnetically alloyed $\mathrm{Sm}_{1-x} \mathrm{M}_{x} \mathrm{~B}_{6}(\mathrm{M}=\mathrm{Ce}$, $\mathrm{Eu}$ ), using angle-resolved photoemission spectroscopy (ARPES) and complementary characterization techniques. Remarkably, topologically nontrivial bulk and surface band structures are found to persist in highly modified samples with up to $30 \%$ Sm substitution, and with an antiferromagnetic ground state in the case of Eu doping. The results are interpreted in terms of a hierarchy of energy scales, in which surface state emergence is linked to the formation of a direct Kondo gap, while low temperature transport trends depend on the indirect gap.
\end{abstract}

The compound $\mathrm{SmB}_{6}$ is a mixed-valence Kondo lattice system [1] that has been under intensive study since 2010 as a strong candidate for the topological Kondo insulator (TKI) state [4]6]. Angle-resolved photoemission (ARPES) measurements of the bulk band structure corroborate a TKI picture, in which dispersive $5 d$-orbital bands hybridize with semi-localized $4 f$ orbital states at the Fermi level to open a gap with topologically inverted symmetries $[7-12$. Topologicallyassociated surface states are seen to emerge as the gap opens at $\mathrm{T} \sim 120 \mathrm{~K}$, and surface conductivity results in a low temperature $(\mathrm{T}<10 \mathrm{~K})$ resistivity plateau. The bulk insulating behavior and surface conductivity are strikingly robust against fractional changes in stoichiometry and non-magnetic alloying [13-15, however doping with magnetic elements $(\mathrm{Ce}, \mathrm{Gd})$ at a far lower $\sim 1 \%$ level eliminates signatures of surface conductivity [13, 14, consistent with the expectation that magnetic disorder will introduce backscattering and Anderson localization to the topological surface state system [16-18]. While these alloys represent fascinating extensions to the physics of the parent compound, Kondo latices are thought to be highly sensitive to disorder 37 39, and the fate of the TKI band structure in alloyed scenarios is unknown. Here, we report a comparative study of the electronic band structure, transport, and magnetic susceptibility properties of alloys incorporating small- and large-moment magnetic lanthanide elements as $\mathrm{Sm}_{1-x} \mathrm{M}_{x} \mathrm{~B}_{6}(\mathrm{M}=\mathrm{Ce}, \mathrm{Eu})$. A clean low-temperature gap in the bulk $5 d$ band is found to persist at the Fermi level up to the highest admixture levels tested $(30 \% \mathrm{Ce}, 20 \% \mathrm{Eu})$, and to be spanned by topologically-associated surface states. The emergence of Fermi level topological surface states in samples with an antiferromagnetic ground state $\left(\mathrm{Sm}_{1-x} \mathrm{Eu}_{x} \mathrm{~B}_{6}, \mathrm{x} \geq 0.1\right)$ presents an antiferromagnetic topological insulator scenario [40, 41] that has been much sought in recent years, with an advantage over other material candidates in that the topological band gap is positioned at the Fermi level. The physical conditions under which topological surface states emerge are found to remain tied to the direct $5 d$ gap, and to allow for a broad range of low temperature resistivity trends defined by the indirect Kondo gap [4244.

Single crystals of $\mathrm{Sm}_{1-x} \mathrm{M}_{x} \mathrm{~B}_{6}(\mathrm{M}=\mathrm{Eu}, \mathrm{Ce})$ were prepared by the alumina flux method, and details of the sample growth are described in the Supplemental Material (SM, Note 1 [18]). Multiple characterization methods, including X-ray diffraction (XRD), scanning tunneling microscope (STM) and ultraviolet X-ray photoemission spectroscopy (UPS) were performed, revealing homogeneous alloying within single-phase crystals with stoichiometry-consistent substitution on the lanthanide site (SM, Note 2 [18). Most ARPES measurements were performed at beamline 4.0.3 at the Advanced Light Source, with a base pressure better than $5 \times 10^{-11}$ Torr. The photon energy was set to $\mathrm{h} \nu=70 \mathrm{eV}$, corresponding to a bulk $\Gamma$-plane of the cubic Brillouin zone (see SM, Note 8 [18, for other photon energies). Energy resolution was $\delta E \lesssim 10 \mathrm{meV}$, and momentum resolution along the dispersive axis of measurement was $\delta k<3 \times 10^{-3} \AA^{-1}$. Measurements of $k_{z}$ dependence for $\mathrm{Sm}_{0.7} \mathrm{Ce}_{0.3} \mathrm{~B}_{6}$ were performed at the NSLS-II ESM beamline, under approx- 

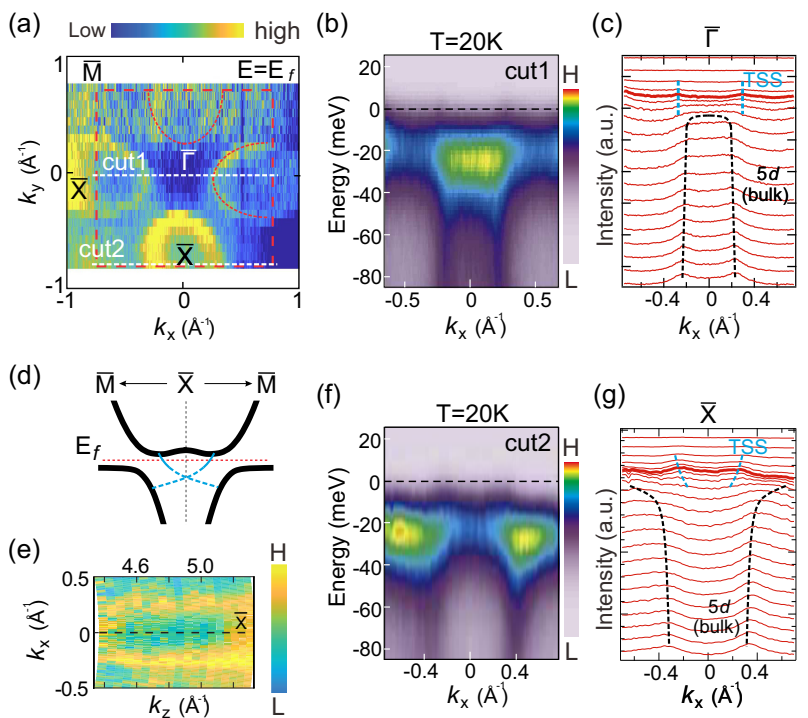

FIG. 1. Surface states after $\mathbf{3 0} \%$ Ce doping. (a) The Fermi surface of $\mathrm{Sm}_{0.7} \mathrm{Ce}_{0.3} \mathrm{~B}_{6}$, with dashed lines tracing ovoid surface state electron pockets. (b) A low-energy ARPES cut along the $\bar{X}-\bar{\Gamma}-\bar{X}$ momentum axis (cut 1). (c) Surface (blue) and bulk (black) bands are traced on momentum distribution curves from panel (b), with an energy step of $6 \mathrm{meV}$. (d) A band structure diagram showing (blue) a topological surface state and (black) hybridization-gapped bulk bands along the $\bar{M}-\bar{X}-\bar{M}$ axis. The lower half of the surface Dirac cone is expected to be a weaker bulk-degenerate resonance state. (e) The two dimensionality of the surface state is visible in $\mathrm{k}_{z}$-dependence of the cut 2 Fermi surface. (f-g) An ARPES cut along the $\bar{M}-\bar{X}-\bar{M}$ direction. The energy step in panel (g) is $5 \mathrm{meV}$. All measurements were performed at $\mathrm{T}=20 \mathrm{~K}$.

imately the same conditions. Samples were cleaved in situ at $\mathrm{T} \sim 20 \mathrm{~K}$, using a top post glued to the (001) surface. All ARPES data were taken within 10 hours after cleavage, and band structure near the Fermi level was stable on this time scale (SM, Note 3 [18). Additional methods details for resistivity and magnetic susceptibility measurements are described in (SM, Note 4 [18]).

There are conflicting interpretations on whether a complete topological classification of $\mathrm{SmB}_{6}$ surface states has been achieved [45 47, particularly with respect to weakly visible Fermi surfaces surrounding the Brillouin zone center. However, measurements consistently show a single surface state Fermi surface surrounding the surface $\bar{X}$-point, with a contour that is not greatly influenced by surface termination [7-12, 45, 47. Spin-resolved investigations have found this state to be singly degenerate [12, 48, 49, creating a topologically nontrivial surface state scenario along the $\bar{X}-\bar{M}$ axis (see Fig. 1(d) diagram, and SM, Note 5 [18]).

High-resolution ARPES experiments were performed on $\mathrm{Sm}_{0.7} \mathrm{Ce}_{0.3} \mathrm{~B}_{6}$ and $\mathrm{Sm}_{0.8} \mathrm{Eu}_{0.2} \mathrm{~B}_{6}$ to address the question of how this topological surface state responds to the altered chemical environments. For $\mathrm{Sm}_{0.7} \mathrm{Ce}_{0.3} \mathrm{~B}_{6}$, low temperature $(\mathrm{T}=20 \mathrm{~K})$ measurements show a four-pocket Fermi surface with a long elliptical orientation along the $\bar{\Gamma}-\bar{X}$ axis, where the Fermi momentum is marginally closer to $\bar{\Gamma}$ than to $\bar{X}$ (Fig. 1(a)). These states are two dimensional (Fig. 1(e); see also Supplementary Note 8 [44]), and qualitatively identical to the $\bar{X}$-point surface state Fermi surface observed at low temperature for undoped $\mathrm{SmB}_{6}$. Fermi pockets surrounding the $\bar{\Gamma}$-point are not seen, as is often the case for undoped $\mathrm{SmB}_{6}$ under the same measurement conditions [10, 11]. Examining band dispersions along the $\bar{\Gamma}-\bar{X}$ axis reveals steeply sloped bulk states (black dashed lines in Fig. 1(c)) from the $\bar{X}$-point $5 d$ pocket, which merge with a flat band associated with $4 f$ states. At the Fermi level, there is no feature at the extrapolated Fermi momentum of the $5 d$ bulk bands $\left(\sim 0.17 \AA^{-1}\right.$ for Fig. $1(\mathrm{c})$, and $\sim 0.41 \AA^{-1}$ for Fig. 1(g)), indicating a well-defined bulk hybridization gap. The gap is crossed by highly dispersive surface states that are offset in momentum from the lower dispersion of the $5 d$ band (see guides to the eye in Fig. 1(c)) as is typically seen for undoped $\mathrm{SmB}_{6}$. A similar scenario is seen along the $\bar{X}-\bar{M}$ axis (Fig. $1(\mathrm{~g})$ ), with only a single surface state intersecting the Fermi level as required for the topological insulator state attributed to $\mathrm{SmB}_{6}$.

The same features are seen in $\mathrm{Sm}_{0.8} \mathrm{Eu}_{0.2} \mathrm{~B}_{6}$ (Fig. 2(ab)), however we find that the bulk electron pocket contours have shifted away from the Brillouin zone center by $\Delta \mathrm{k}=0.07 \AA^{-1}$ relative to $\mathrm{Sm}_{0.7} \mathrm{Ce}_{0.3} \mathrm{~B}_{6}$. If taken to represent an isotropic fractional change in Fermi momentum, this indicates the addition of 0.2 holes per unit cell in the $5 d$ orbital. This difference in the bulk electronic structures can be understood by noting that the samarium sites in $\mathrm{SmB}_{6}$ are mixed-valent with a roughly equal mixture of $4 f^{5}$ and $4 f^{6}$ configurations [50], whereas cerium and europium have strongly favored $f$-shell occupancies of $4 f^{1}\left(\mathrm{Ce}^{3+}\right)$ and $4 f^{7}\left(\mathrm{Eu}^{2+}\right)$ [51, 52] (see characterization in SM, Note 6 [18]), and can be expected to contribute $\sim 50 \%$ fractional electron $\left(\mathrm{Ce}^{3+}\right)$ and hole $\left(\mathrm{Eu}^{2+}\right)$ doping, respectively, to the samarium sublattice. The observed difference in bulk $5 d$ dispersions accounts for roughly $80 \%$ of this nominal doping effect, suggesting that most of the doped charge resides in itinerant $5 d$ states, with just a few percent $(<\sim 5 \%)$ of an electron or hole doped into the strongly correlated $\mathrm{Sm} 4 f$ sublattice.

Raising the temperature of $\mathrm{Sm}_{0.8} \mathrm{Eu}_{0.2} \mathrm{~B}_{6}$ from $10 \mathrm{~K}$ to $180 \mathrm{~K}$ shows incremental broadening and a downward energetic shift of the flat $4 f$-state (Fig. 2(b)), as is also seen for undoped $\mathrm{SmB}_{6}[\underline{8}, 11$. The Fermi momentum of the in-gap feature declines at $\mathrm{T}>120 \mathrm{~K}$ (see Fig. 2(b)), as the surface state (with larger Fermi momentum) vanishes and is replaced within the incoherent Kondo gap by a continuation of the bulk $5 d$ state (see Fig. 2(e) progression). The similarity of the low-temperature Fermi level band structure in these highly doped configurations is remarkable, but consistent with recent theory for mixed-valent systems [53, and with a majority of doped electrons oc- 


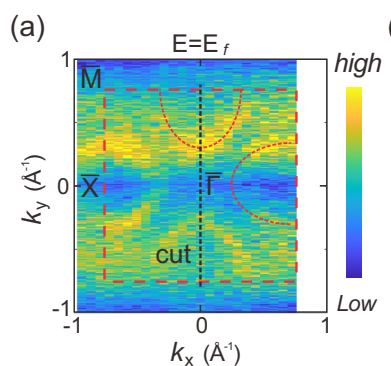

(c)

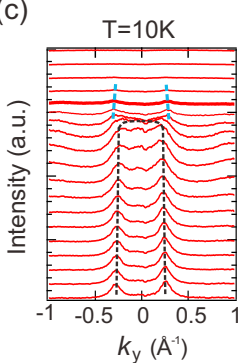

(b)

(d)

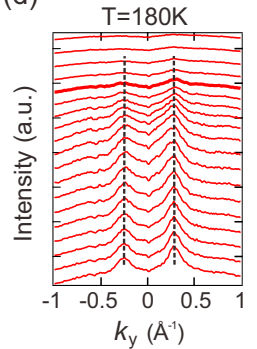

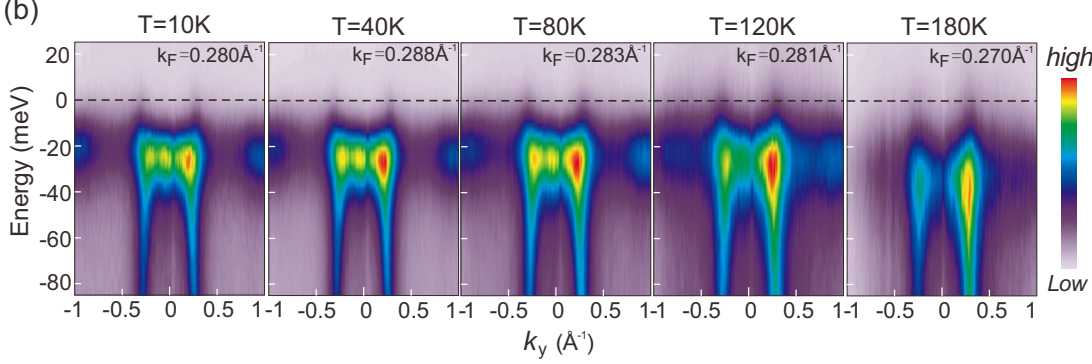

(e)

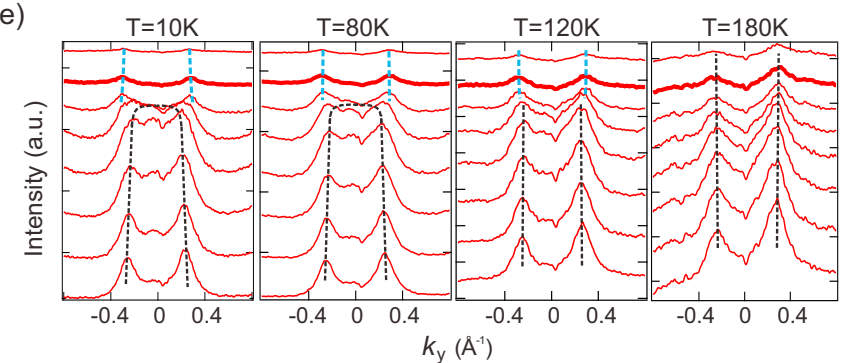

FIG. 2. Surface state emergence after $\mathbf{2 0} \%$ Eu doping. (a) The Fermi surface of $\mathrm{Sm}_{0.8} \mathrm{Eu}_{0.2} \mathrm{~B}_{6}$. (b) A low-energy ARPES cut taken along the high symmetry $\bar{X}-\bar{\Gamma}-\bar{X}$ direction (traced in (a)) is shown as a function of temperature, and annotated with the momentum-axis center of mass of Fermi level features. Raw data underlying the $\mathrm{T}=10 \mathrm{~K}$ (c) and $180 \mathrm{~K}$ (d) images are traced with surface (blue) and bulk (black) bands. The Fermi level is indicated with a thicker line, and the energy step is $6 \mathrm{meV}$. (e) Raw data curves within $50 \mathrm{meV}$ of the Fermi level are shown for selected temperatures, with an energy step of $7 \mathrm{meV}$.

cupying the itinerant $5 d$ states as attributed above.

Unlike these topological features, local moment physics and many-body ordering energetics cannot be unaffected by magnetic doping. As Ce doping level increases, the magnetic susceptibility $(\chi-\mathrm{T})$ curves evolve rapidly and nonlinearly to strongly resemble the $\mathrm{CeB}_{6}$ curve after just $30 \%$ substitution (Fig. 3(a)). Fitting inverse susceptibility $(1 / \chi-T)$ with a Curie-Weiss function in the hightemperature paramagnetic regime (SM, Note 7 [18]) reveals effective local moments of $5.4 \mu_{B}\left(\mathrm{Sm}_{0.99} \mathrm{Ce}_{0.01} \mathrm{~B}_{6}\right)$, $5.15 \mu_{B}\left(\mathrm{Sm}_{0.97} \mathrm{Ce}_{0.03} \mathrm{~B}_{6}\right), 4.2 \mu_{B}\left(\mathrm{Sm}_{0.9} \mathrm{Ce}_{0.1} \mathrm{~B}_{6}\right), 3.15 \mu_{B}$ $\left(\mathrm{Sm}_{0.7} \mathrm{Ce}_{0.3} \mathrm{~B}_{6}\right)$. The terminal value at $30 \%$ doping is remarkably close to the local moment of $\mathrm{CeB}_{6}$, or the free-ion $4 f^{1}$ scenario, both of which are around $2.54 \mu_{B}$ [54, 55].

Large-moment doping with Eu yields a similarly nonlinear trend, but in the opposite direction. Europium gravitates strongly to the half-filled large spin $4 f^{7}$ configuration favored by intra-atomic exchange interactions, with a large effective moment of $8 \mu_{B}$ in $\mathrm{EuB}_{6}$ [56. Partial substitution of samarium as $\mathrm{Sm}_{1-x} \mathrm{M}_{x} \mathrm{~B}_{6}$ induces antiferromagnetic order [57, which can be seen from the susceptibility kinks at $\mathrm{T}=2.8 \mathrm{~K}\left(\mathrm{Sm}_{0.9} \mathrm{Eu}_{0.1} \mathrm{~B}_{6}\right)$ and $\mathrm{T}=7 \mathrm{~K}\left(\mathrm{Sm}_{0.8} \mathrm{Eu}_{0.2} \mathrm{~B}_{6}\right)$ in Fig. 3(b). The effective moments extracted from inverse susceptibility for Eu-alloyed $\mathrm{SmB}_{6}$ are $4.2 \mu_{B}\left(\mathrm{Sm}_{0.95} \mathrm{Eu}_{0.05} \mathrm{~B}_{6}\right), \quad 7.1 \mu_{B}$ $\left(\mathrm{Sm}_{0.9} \mathrm{Eu}_{0.1} \mathrm{~B}_{6}\right)$ and $6.1 \mu_{B}\left(\mathrm{Sm}_{0.8} \mathrm{Eu}_{0.2} \mathrm{~B}_{6}\right)$. The rapid change in local moments may be aided by a complementary effect from charge doping. Electron doping from Ce is expected to reduce local moment by promoting occupancy of the zero-moment $\left({ }^{7} \mathbf{F}_{0}\right)$ multiplet ground state of $4 f^{6} \mathrm{Sm}$, and hole doping from Eu will have the opposite effect by biasing the system towards large-moment $4 f^{5}$ configurations.

Transport trends are also very different between the Ce- and Eu-alloyed samples. The resistivity of undoped$\mathrm{SmB}_{6}$ is strikingly enhanced beneath the Kondo onset of T $50 \mathrm{~K}$ (Fig. 3(c)), before flattening into a plateau associated with surface state conductivity [13, 58, 59] at $\mathrm{T}<10 \mathrm{~K}$. Substituting $3 \% \mathrm{Sm}$ with Ce causes $\mathrm{T} \lesssim 5 \mathrm{~K}$ bulk resistivity to be reduced by one to two orders of magnitude (Fig. 3(c)), an effect that seems counterintuitive in the context of adding defects, but matches expectations for an insulating Kondo lattice, as impurities may create in-gap states and suppress the coherence of the insulating Kondo band structure [39, 60]. The Kondoregime resistivity of $\mathrm{Sm}_{1-x} \mathrm{Ce}_{x} \mathrm{~B}_{6}$ is suppressed further at higher doping levels, but retains an upturn beneath $\mathrm{T} \lesssim 50 \mathrm{~K}$. For $\mathrm{Sm}_{0.7} \mathrm{Ce}_{0.3} \mathrm{~B}_{6}$, the trend beneath $\mathrm{T} \lesssim 4 \mathrm{~K}$ appears to be metallic (positively sloped), and resembles pure $\mathrm{CeB}_{6}$ (see Fig. 3(c) inset).

The insulating character of $\mathrm{Sm}_{1-x} \mathrm{Eu}_{x} \mathrm{~B}_{6}$ (Fig. 3(d)) is far more robust, with a non-monotonic trend under doping that has been noted in previous literature [57]. The alloys retain a characteristic Kondo insulating exponential trend (see SM, Fig. S9 18). Mild inflections of resistivity are seen at the Néel transitions, but are not very distinguishable from other nonlinear details within the 
(a)

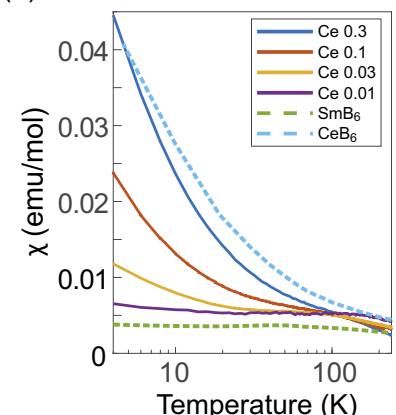

(b)

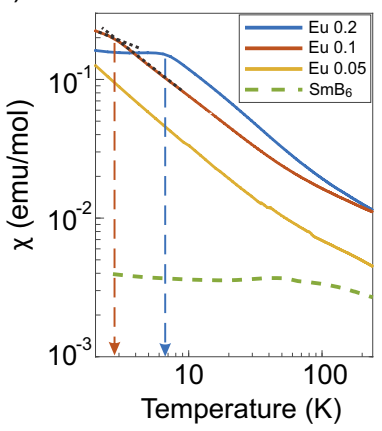

(c)

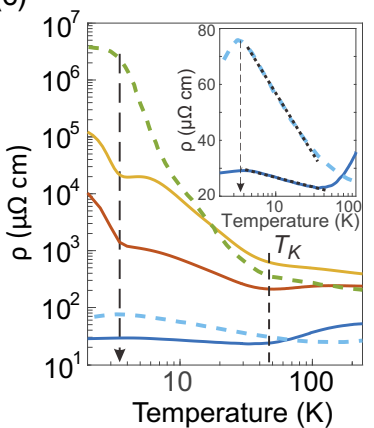

(d)

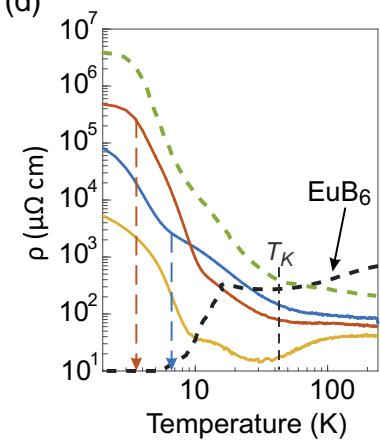

FIG. 3. Resistivity and susceptibility of magnetically doped alloys. (a)Magnetic susceptibility of Ce-alloyed $\mathrm{SmB}_{6}$. Data for pristine $\mathrm{CeB}_{6}$ and $\mathrm{SmB}_{6}$ are extracted from refs. [55] and 67. (b) Magnetic susceptibility of Eu-alloyed $\mathrm{SmB}_{6}$. Drop-arrows mark the magnetic transition at $10 \%$ and $20 \%$ Eu doping levels. (c) Resistivity of Ce-alloyed $\mathrm{SmB}_{6}$. A black drop-arrow marks the onset of the $\mathrm{T}<10 \mathrm{~K}$ surface conductivity plateau for $\mathrm{SmB}_{6}$, which happens to coincide with bulk-derived resistivity kinks in the alloyed samples. Data for pristine $\mathrm{CeB}_{6}$ and $\mathrm{SmB}_{6}$ are extracted from ref. 68 and ref. 69, and an inset compares the resistivity trends for $\mathrm{CeB}_{6}$ and $\mathrm{Sm}_{0.7} \mathrm{Ce}_{0.3} \mathrm{~B}_{6}$. (d) Resistivity of Eu-alloyed $\mathrm{SmB}_{6}$, with data for pristine $\mathrm{EuB}_{6}$ extracted from ref. [56]. Red and blue arrows indicate magnetic transitions, as in panel (b).

plot, and differ starkly from the dramatic change caused by ferromagnetism at $\mathrm{T}_{C} \sim 12 \mathrm{~K}$ in $\mathrm{EuB}_{6}$ [56].

With respect to the trend toward metallicity in Ce- alloys, it is intriguing to note that pure $\mathrm{CeB}_{6}$ also presents a $4 f / 5 d$ Kondo lattice scenario, with a superficially similar onset of Kondo-associated resistivity to $\mathrm{SmB}_{6}$ at $\mathrm{T} \lesssim 50 \mathrm{~K}$ (Fig. 3(c)). However, the $\mathrm{CeB}_{6}$ bulk band structure that emerges from low-temperature Kondo coherence is metallic due to the high density of Kondoactive large-moment sites [61, and the modest increase of resistivity as Kondo coherence sets in appears to represent the transition to a low-mobility heavy Fermion band structure $[62,63]$. However, there is no fully established approach to modeling the band structure of a dense Kondo lattice like $\mathrm{CeB}_{6}$, or a mixed-valent one like $\mathrm{SmB}_{6}$. Theory for $\mathrm{SmB}_{6}$ often builds from a computationally tractable single-particle band hybridization picture, however the monovalent nature ascribed to cerium

in $\mathrm{CeB}_{6}$ is much less compatible with such a picture, and the coincidence of Kondo temperature scales within resistivity measurements is suggestive that a complete theory for $\mathrm{Sm}_{0.7} \mathrm{Ce}_{0.3} \mathrm{~B}_{6}$ may need to go beyond a single-particle modeling basis.

From an empirical perspective, $\mathrm{Sm}_{0.7} \mathrm{Ce}_{0.3} \mathrm{~B}_{6}$ has superior $\mathrm{T}<50 \mathrm{~K}$ conductivity to pure $\mathrm{CeB}_{6}$ and preserves the topological surface state of $\mathrm{SmB}_{6}$, and so appears to be a poor fit for the standard picture in which the metallicity of a disordered Kondo insulator comes from an in-gap impurity band [39. Examining the low temperature $4 f$ states in greater detail, we find that they are broader along the energy axis than those seen in $\mathrm{SmB}_{6}$, with a peak width at half maximum (half-width) that is greater by $\sim 15-20 \mathrm{meV}$ (Fig. 4(a)). This change is much larger than the $\sim 4 \mathrm{meV}$ insulating gap attributed from the activation of resistivity in $\mathrm{SmB}_{6}$ [15], and suggests that impurities may close the gap by introducing a broader continuum of diffusively conducting states. Similar $4 f$-associated states are seen by ARPES at low temperature in $\mathrm{CeB}_{6}$, but are much broader [64, 65], making it difficult to perform a fine comparison.

As temperature increases, a rapid thermal activation of $4 f$ half-width is seen for undoped $\mathrm{SmB}_{6}$ and $\mathrm{Sm}_{0.8} \mathrm{Eu}_{0.2} \mathrm{~B}_{6}$ at $\mathrm{T} \gtrsim 80 \mathrm{~K}$, and is consistent with the square of temperature $\left(\mathrm{T}^{2}\right)$ trend expected for Fermi liquid self-energy. In all cases, surface states and $4 f / 5 d$ hybridization are visible at $4 f$ half-width values less than 40 $\mathrm{meV}$, found at temperatures $\mathrm{T} \lesssim 120 \mathrm{~K}$. This phenomenology is consistent with recent theoretical investigations, which have proposed that the topological insulating gap of band insulator-like TKI systems may be highly robust against defects at the $\sim 20 \%$ level 53 , 66. This picture may also relate to the lack of surface states in ARPES studies of pure $\mathrm{CeB}_{6}$ 64, 65, which has a $4 f$-state width considerably larger than $40 \mathrm{meV}$.

The picture that emerges is one in which the topological surface states of $\mathrm{SmB}_{6}$ alloys begin to emerge in concert with the direct $4 f / 5 d$ hybridization gap as coherence improves at $\mathrm{T} \lesssim 120 \mathrm{~K}$. This matches expectations that the direct Kondo gap will define topology within a band picture (see Fig. 4(c)). The transition is unlikely to be sudden, as earlier studies on undoped $\mathrm{SmB}_{6}$ have shown that the bulk bands evolve continuously throughout the onset of f-electron coherence [11. Surface states satisfy a nontrivial topology along the $\mathrm{X}-\mathrm{M}$ axis, and are found near the outer boundary of the $\mathrm{k}_{z}$-projected $5 d$ state continuum (Fig. 4(b)). The robustness of both surface states and the gap are remarkable given that $\mathrm{SmB}_{6}$ may otherwise be unique as a TKI, and may be due to the unusual tendency of Sm towards mixed-valent electronic configurations [53, 66] (see further discussion at the end of SM, Note 2 [18]). Moreover, the crossover to a metallic (positively sloped) low temperature resistivity trend appears to relate to changes in the band structure on a smaller energy scale comparable to the $\sim 4 \mathrm{meV}$ indirect 
band gap.

Taken collectively, these results show that TKI surface states can still emerge in alloys that deviate greatly from the parent compound $\mathrm{SmB}_{6}$ in terms of metallicity, magnetic local moment, and magnetic ground state. This will enable the exploration of TKI surface physics in new physical regimes, and supports recent theoretical modeling, which has suggested that topological band gap and surface state properties of $\mathrm{SmB}_{6}$-like TKI materials will be far more robust against disorder than is typically expected for Kondo lattice systems [53, 66. Comparing these alloys with undoped $\mathrm{SmB}_{6}$ and $\mathrm{CeB}_{6}$, the emergence of TKI surface states is found to consistently coincide with the opening of a direct band gap in the bulk $5 d$ spectral function, which in turn is associated with $4 f$-state half-widths falling beneath a critical $\lesssim 40 \mathrm{meV}$ threshold. Local moments are seen to vary by a factor of two, from roughly $3.15 \mu_{B}$ in $\mathrm{Sm}_{0.7} \mathrm{Ce}_{0.3} \mathrm{~B}_{6}$ to over $6 \mu_{B}$ in $\mathrm{Sm}_{1-x} \mathrm{Eu}_{x} \mathrm{~B}_{6}(\mathrm{x}=0.1,0.2)$, and the differing $4 f$-electron energetics appear to result in contrasting trends towards metallic-like and insulating-like Kondo lattice scenarios for Ce- and Eu-alloys, respectively. The insulating-like scenario realized by $\mathrm{Sm}_{0.8} \mathrm{Eu}_{0.2} \mathrm{~B}_{6}$ places topological surface states at the Fermi level in a system with $\mathrm{T}_{N}=7 \mathrm{~K}$ antiferromagnetism, presenting a remarkably clean candidate system for the much-sought antiferromagnetic TI state.

\section{ACKNOWLEDGEMENTS}

Spectroscopic measurements in the main text were performed at the Advanced Light Source, supported by the Director, Office of Science, Office of Basic Energy Sciences, of the U.S. Department of Energy under Contract No. DE-AC02-05CH11231. Operation of the ESM beamline at the National Synchrotron Light Source is supported by DOE Office of Science User Facility Program operated for the DOE Office of Science by Brookhaven National Laboratory under Contract no. DE-AC0298CH10886. Work at NYU was supported by the MRSEC Program of the National Science Foundation under Grant Number DMR-1420073. The STM work at Rutgers is supported by NSF under grant DMR-1506618. C.-H. Min, K. Kißner, and F. Reinert acknowledge financial support from the DFG through SFB1170 'tocotronics' and the Würzburg-Dresden Cluster of Excellence on Complexity and Topology in Quantum Matter - ct.qmat (EXC 2147, project-id 39085490). M.S. Song, B.Y. Kang and B. K. Cho were supported by National Research Foundation of Korea (NRF), funded by the Ministry of Science, ICT \& Future Planning (No. NRF-2017R1A2B2008538). L. Miao is supported by the National Natural Science Foundation of China (Grants No. U2032156 and No. 12004071) and Natural Science Foundation of Jiangsu Province, China (Grant No.

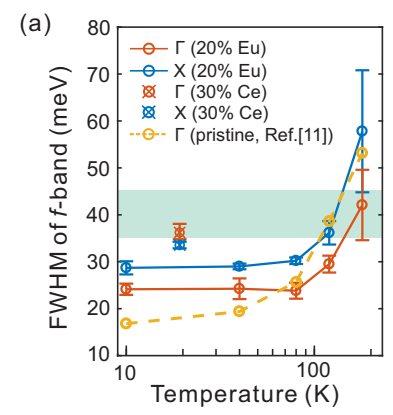

(b)
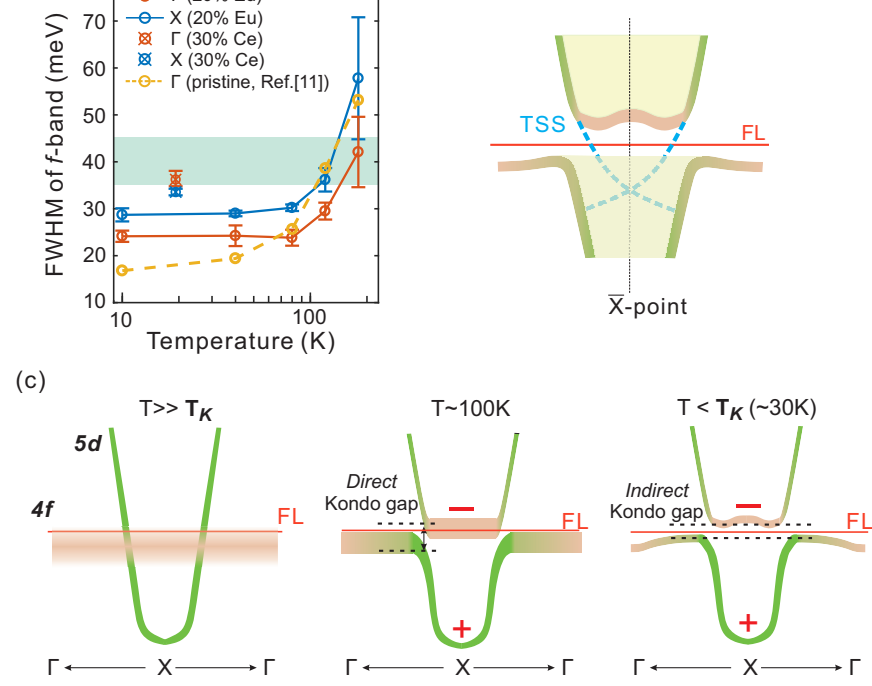

FIG. 4. Coherence, hybridization and surface state emergence. (a) The $4 f$ band feature width at half maximum in alloys is evaluated from a Voigt fit and compared with pristine $\mathrm{SmB}_{6}$ as a function of temperature. A shaded region indicates the threshold below which surface states become visible to ARPES. (b) A schematic showing surface states spanning the hybridization gap. Bulk states with zero z-axis momentum are drawn as solid lines, and the $\mathrm{k}_{z}$-projected bulk state continuum is shown with light yellow shading. (c) Diagrams show the temperature-resolved evolution of TKI bulk band structure intersecting the X-point. (middle) As coherence increases with the lowering of temperature, topologically inverted direct hybridization gaps open at band crossing points. (right) As temperature decreases further, the presence or absence of an indirect gap within the Kondo band structure defines the trend of resistivity. The topologically inverted parity symmetries are indicated by ' + ' ( $d$-orbital) and '-' $(f$-orbital) signs

\section{BK20200348).}

* Electronic address: lawray@nyu.edu Corresponding author

[1] A. Menth, E. Buehler, and T.H. Geballe, Magnetic and Semiconducting Properties of $\mathrm{SmB}_{6}$, Phys. Rev. Lett. 22, 295 (1969).

[2] R.L. Cohen, M. Eibschütz, and K.W. West, Electronic and Magnetic Structure of $\mathrm{SmB}_{6}$,Phys. Rev. Lett. 24, 383 (1970).

[3] M. Dzero, J. Xia, V. Galitski, and P. Coleman, Topological Kondo Insulators, Annu. Rev. Condens. Matter Phys. 7, 249 (2016).

[4] M. Dzero, K. Sun, V. Galitski, and P. Coleman, Topological Kondo Insulators, Phys. Rev. Lett. 104, 106408 (2010).

[5] M. Dzero, K. Sun, P. Coleman, and V. Galitski, Theory of topological Kondo insulators, Phys. Rev. B 85, 045130 (2012).

[6] F. Lu, J. Z. Zhao, H. Weng, Z. Fang, and X. Dai, Corre- 
lated Topological Insulators with Mixed Valence, Phys. Rev. Lett. 110, 096401 (2013).

[7] M. Neupane, N. Alidoust, S-Y. Xu, T. Kondo, Y. Ishida, D. J. Kim, C. Liu, I. Belopolski, Y. J. Jo, T-R. Chang, H-T. Jeng, T. Durakiewicz, L. Balicas, H. Lin, A. Bansil, S. Shin, Z. Fisk and M. Z. Hasan, Surface electronic structure of the topological Kondo-insulator candidate correlated electron system $\mathrm{SmB}_{6}$, Nat. Commun. 4, 2991 (2013).

[8] J. Jiang, S. Li, T. Zhang, Z. Sun, F. Chen, Z.R. Ye, M. Xu, Q.Q. Ge, S.Y. Tan, X.H. Niu, M. Xia, B.P. Xie, Y.F. Li, X.H. Chen, H.H. Wen and D.L. Feng, Observation of possible topological in-gap surface states in the Kondo insulator $\mathrm{SmB}_{6}$ by photoemission, Nat. Commun. 4, 3010 (2013).

[9] N. Xu, X. Shi, P. K. Biswas, C. E. Matt, R. S. Dhaka, Y. Huang, N. C. Plumb, M. Radović, J. H. Dil, E. Pomjakushina, K. Conder, A. Amato, Z. Salman, D. McK. Paul, J. Mesot, H. Ding, and M. Shi, Surface and bulk electronic structure of the strongly correlated system $\mathrm{SmB}_{6}$ and implications for a topological Kondo insulator, Phys. Rev. B 88, 121102(R). (2013).

[10] J. D. Denlinger, J. W. Allen, J-S. Kang, K. Sun, B-I. Min, D.-J. Kim, and Z. Fisk, $\mathrm{SmB}_{6}$ Photoemission: Past and Present, JPS Conf. Proc. 3, 017038 (2014).

[11] J. D. Denlinger, J. W. Allen, J.-S. Kang, K. Sun, J.-W. Kim, J. H. Shim, B. I. Min, D.-J. Kim, and Z. Fisk, Temperature Dependence of Linked Gap and Surface State Evolution in the Mixed Valent Topological Insulator $\mathrm{SmB}_{6}$, arXiv:1312.6637 (2014).

[12] Y. Ohtsubo, Y. Yamashita, K. Hagiwara, S. Ideta, K. Tanaka, R. Yukawa, K. Horiba, H. Kumigashira, K. Miyamoto, T. Okuda, W. Hirano, F. Iga and S. Kimura, Non-trivial surface states of samarium hexaboride at the (111) surface, Nat. Commun. 10, 2298 (2019).

[13] D.-J. Kim, J. Xia and Z. Fisk, Topological surface state in the Kondo insulator samarium hexaboride, Nat. Mater. 13, 466 (2014).

[14] B. Y. Kang, C.-H. Min, S. S. Lee, M. S. Song, K. K. Cho, and B. K. Cho, Magnetic and nonmagnetic doping dependence of the conducting surface states in $\mathrm{SmB}_{6}$, Phys. Rev. B 94, 165102 (2016).

[15] Y. S. Eo, A. Rakoski, J. Lucien, D. Mihaliov, C. Kurdak, P. F. S. Rosa, and Z Fisk, Transport gap in $\mathrm{SmB}_{6}$ protected against disorder, Proc. Natl. Acad. Sci. U. S. A. 116, 12638 (2019).

[16] X.-L. Qi and S.-C. Zhang, Topological insulators and superconductors, Colloquium: Topological insulators, Rev. Mod. Phys. 83, 1057 (2011).

[17] M. Z. Hasan and C. L. Kane, Rev. Mod. Phys. 82, 3045 (2010).

[18] See Supplemental Material for additional technique and characterization details, which includes Refs. [19]36].

[19] J. M. Lawrence et al., Kondo hole behavior in $\mathrm{Ce}_{0.97} \mathrm{La}_{0.03} \mathrm{Pd}_{3}$, Phys. Rev. B 53, 12559 (1996).

[20] M. H. Hamidian et al., How Kondo-holes create intense nanoscale heavy-fermion hybridization disorder, Proc. Natl. Acad. Sci. USA 108, 18233-18237 (2011).

[21] P. F. S. Rosa, A. Oostra, J. D. Thompson, P. G. Pagliuso, and Z. Fisk, Unusual Kondo-hole effect and crystal-field frustration in Nd-doped $\mathrm{CeRhIn}_{5}$, Phys. Rev. B 94, 045101 (2016).

[22] H. He et al., Irreversible proliferation of magnetic moments at cleaved surfaces of the topological Kondo insu- lator $\mathrm{SmB}_{6}$, Phys. Rev. B 95, 195126 (2017).

[23] P. Lutz et al., Valence characterisation of the subsurface region in $\mathrm{SmB}_{6}$, Philos. Mag. 96, 3307 (2016).

[24] S. Suga et al., Spin-Polarized Angle-Resolved Photoelectron Spectroscopy of the So-Predicted Kondo Topological Insulator $\mathrm{SmB}_{6}$. J. Phys. Soc. Jpn. 83, 014705 (2014).

[25] L. Fu, C. L. Kane, and E. J. Mele, Topological Insulators in Three Dimensions, Phys. Rev. Lett. 98, 106803 (2007).

[26] F. Siek et al., Angular momentum?induced delays in solid-state photoemission enhanced by intra-atomic interactions, Science 357, 1274 (2017).

[27] S. Rößler, et al., Hybridization gap and Fano resonance in $\mathrm{SmB}_{6}$, Proc. Nat. Acad. Sci. 111, 4798 (2014).

[28] C. E. Matt et al., Consistency between ARPES and STM measurements on $\mathrm{SmB}_{6}$, Phys. Rev. B 101, 085142 (2020).

[29] J. A. Colón Santana et al., Effect of gadolinium doping on the electronic band structure of europium oxide, Phys. Rev. B 85, 014406 (2012).

[30] Hartree-Fock Slater Condon parameters were obtained from the Cowan code (see https://www.tcd.ie/Physics/people/Cormac.McGuinness/Cowan/)

[31] E. Anderson, et al., LAPACK User?s Guide, 3rd ed. (SIAM, Philadelphia, 1999).

[32] A. Maurya, R. Kulkarni, A. Thamizhavel, D. Paudyal, and S. K. Dhar, Kondo lattice and antiferromagnetic behavior in quaternary $\mathrm{CeTAl}_{4} \mathrm{Si}_{2}(\mathrm{~T}=\mathrm{Rh}, \mathrm{Ir})$ single crystals, J. Phys. Soc. Jpn. 85, 034720 (2016).

[33] Y. Singh, R. S. Sannabhadti, and S. Ramakrishnan, Lowtemperature properties of a new Kondo Lattice compound $\mathrm{Yb}_{2} \mathrm{Ir}_{3} \mathrm{Sn}_{5}$, arXiv:cond-mat/0510583

[34] F. Gerken, Calculated photoemission spectra of the $4 \mathrm{f}$ states in the rare-earth metals, J. Phys. F: Met. Phys. 13, 703 (1983)

[35] M. Liu et al., Crossover between Weak Antilocalization and Weak Localization in a Magnetically Doped Topological Insulator, Phys. Rev. Lett. 108, 036805 (2012)

[36] Y. Xu et al., Disorder enabled band structure engineering of a topological insulator surface, Nat. Commun. 8, 14081 (2017).

[37] J. D. Thompson, Holes in a Kondo lattice, Proc. Natl. Acad. Sci. USA 108, 18191 (2011).

[38] R. Sollie and P. Schlottmann, A simple theory of the Kondo hole, J. Appl. Phys. 69, 5478-5480 (1991).

[39] P. Schlottmann and C. S. Hellberg, Metal-insulator transition in dirty Kondo insulators, J. Appl. Phys. 79, 6414 (1996).

[40] R. S. K. Mong, A. M. Essin, and J. E. Moore, Antiferromagnetic topological insulators, Phys. Rev. B 81, 245209 (2010).

[41] J. Li, Y. Li, S. Du, Z. Wang, B.-L. Gu, S.-C. Zhang, K. He, W. Duan, and Y. Xu, Intrinsic magnetic topological insulators in van der Waals layered $\mathrm{MnBi}_{2} \mathrm{Te}_{4}$-family materials, Sci. Adv. 5, eaaw5685 (2019).

[42] C. Sanchez-Castro, P. Bedell and B. Cooper, Hybridization gap insulating behavior of $\mathrm{Ce}_{3} \mathrm{Bi}_{4} \mathrm{Pt}_{3}$, Phys. Rev. B 47, 6879 (1993).

[43] B. Bucher, Z. Schlesinger, P. C. Canfield and Z. Fisk, Kondo coupling induced charge gap in $\mathrm{Ce}_{3} \mathrm{Bi}_{4} \mathrm{Pt}_{3}$, Phys. Rev. Lett. 72, 522 (1994).

[44] N.S. Vidhyadhiraja and D. E. Logan, Optical and transport properties of heavy fermions, J. Phys.: Condens. Matter 17, 2959 (2005).

[45] Z.-H. Zhu, A. Nicolaou, G. Levy, N. P. Butch, P. Syers, 
X. F. Wang, J. Paglione, G. A. Sawatzky, I. S. Elfimov, and A. Damascelli, Polarity-Driven Surface Metallicity in $\mathrm{SmB}_{6}$, Phys. Rev. Lett. 111, 216402 (2013).

[46] E. Frantzeskakis, N. de Jong, B. Zwartsenberg, Y. K. Huang, Y. Pan, X. Zhang, J. X. Zhang, F. X. Zhang, L. H. Bao, O. Tegus, A. Varykhalov, A. de Visser, and M. S. Golden, Kondo Hybridization and the Origin of Metallic States at the (001) Surface of $\mathrm{SmB}_{6}$, Phys. Rev. X 3, 041024 (2013).

[47] P. Hlawenka, K. Siemensmeyer, E. Weschke, A. Varykhalov, J. Sánchez-Barriga, N. Y. Shitsevalova, A. V. Dukhnenko, V. B. Filipov, S. Gabáni, K. Flachbart, O. Rader and E. D. L. Rienks, Samarium hexaboride is a trivial surface conductor, Nat. Commun. 9, 517 (2018).

[48] N. Xu, P. K. Biswas, J. H. Dil, R. S. Dhaka, G. Landolt, S. Muff, C. E. Matt, X. Shi, N. C. Plumb, M. Radović, E. Pomjakushina, K. Conder, A. Amato, S. V. Borisenko, R. Yu, H.-M. Weng, Z. Fang, X. Dai, J. Mesot, H. Ding and M. Shi, Direct observation of the spin texture in $\mathrm{SmB}_{6}$ as evidence of the topological Kondo insulator, Nat. Commun. 5, 4566 (2014).

[49] N. Xu, H. Ding and M. Shi, Spin- and angle-resolved photoemission on the topological Kondo insulator candidate: $\mathrm{SmB}_{6}$, J. Phys. Cond. Matter, 28, 363001 (2016).

[50] J. C. Nickerson, R. M. White, K. N. Lee, R. Bachmann, T. H. Geballe, and G. W. Hull, Jr., Physical Properties of $\mathrm{SmB}_{6}$, Phys. Rev. B 3, 2030 (1971).

[51] M. Magnuson, S. M. Butorin, J.-H. Guo, A. Agui, J. Nordgren, H. Ogasawara, A. Kotani, T. Takahashi, and S. Kunii, Electronic-structure investigation of $\mathrm{CeB}_{6}$ by means of soft-x-ray scattering, Phys. Rev. B 63, 075101 (2001).

[52] Y. Takakuwa, S. Suzuki and T. Sagawa, Photoemission Measurements of EuB 6 , Jpn. J. Appl. Phys.17, 284 (1978).

[53] B. Skinner, Properties of the donor impurity band in mixed valence insulators, Phys. Rev. Mater. 3, 104601 (2019).

[54] S. Blundell, Magnetism in Condensed Matter, Oxford University Press, Oxford. (ISBN: 9780198505914) (2001).

[55] C. Terzioglu, D. A. Browne, R. G. Goodrich, A. Hassan and Z. Fisk, EPR and magnetic susceptibility measurements on $\mathrm{CeB}_{6}$, Phys. Rev. B 63, 235110 (2001).

[56] S. Süllow, I. Prasad, M. C. Aronson, J. L. Sarrao, Z. Fisk, D. Hristova, A. H. Lacerda, M. F. Hundley, A. Vigliante and D. Gibbs, Structure and magnetic order of $\mathrm{EuB}_{6}$, Phys. Rev. B 57, 5860 (1998).

[57] S. Yeo, K. Song, N. Hur, Z. Fisk and P. Schlottmann, Effects of Eu doping on $\mathrm{SmB}_{6}$ single crystals, Phys. Rev. B 85, 115125 (2012).
[58] S. Wolgast, C. Kurdak, K. Sun, J. W. Allen, D.-J. Kim, and Z. Fisk, Low-temperature surface conduction in the Kondo insulator $\mathrm{SmB}_{6}$, Phys. Rev. B 88, 180405(R) (2013).

[59] D. J. Kim, S. Thomas, T. Grant, J. Botimer, Z. Fisk, and Jing Xia, Surface Hall Effect and Nonlocal Transport in $\mathrm{SmB}_{6}$ : Evidence for Surface Conduction, Sci. Rep. 3, 3150 (2013).

[60] S. Wermbter, K. Sabel, and G. Czycholl, Electrical resistivity of heavy-fermion systems with nonmagnetic impurities, Phys. Rev. B 53, 2528 (1996).

[61] J. M. Effantin, J. Rossat-Mignod, and P. Burlet, Magnetic phase diagram of $\mathrm{CeB}_{6}$, J. Magn. \& Magn. Mater., 47-48, 145-148 (1985).

[62] S. Nakatsuji, D. Pines, and Z. Fisk, Two Fluid Description of the Kondo Lattice, Phys. Rev. Lett. 92, 016401 (2004).

[63] P. Coleman, "Heavy Fermions and the Kondo Lattice: a 21st Century Perspective" in Many-Body Physics: From Kondo to Hubbard, E. Pavarini, E. Koch and P. Coleman Eds. (Forschungszentrum Julich, 2015), Chapter 1, 1.11.34

[64] M. Neupane, N. Alidoust, I. Belopolski, G. Bian, S.-Y. Xu, D.-J. Kim, P. P. Shibayev, D. S. Sanchez, H. Zheng, T.-R. Chang, H.-T. Jeng, P. S. Riseborough, H. Lin, A. Bansil, T. Durakiewicz, Z. Fisk, and M. Zahid Hasan, Fermi surface topology and hot spot distribution in the Kondo lattice system CeB 6 , Phys. Rev. B 92, 104420 (2015).

[65] S. V. Ramankuttya, N. de Jonga, Y. K. Huanga, B. Zwartsenberga, F. Massee b, T. V. Baya, M. S. Goldena, and E. Frantzeskakis, Comparative study of rare earth hexaborides using high resolution angle-resolved photoemission, J. Electron Spectrosc. Relat. Phenom. 208, 4350 (2016).

[66] P. P. Baruselli and M. Vojta, Kondo holes in topological Kondo insulators: Spectral properties and surface quasiparticle interference, Phys. Rev. B 89, 205105 (2014).

[67] M. C. Hatnean, M. R. Lees, D. M. Paul, and P. G. Balakrishana, Large, high quality single-crystals of the new Topological Kondo Insulator, $\mathrm{SmB}_{6}$, Sci. Rep. 3, 3071(2013).

[68] T. Fujita, M. Suzuki, T. Komatsubara, S. Kunii, T. Kasuya, and T. Ohtsuka, Anomalous specific heat of $\mathrm{CeB}_{6}$, Solid State Commun. 35, 569 (1980).

[69] X. Zhang, N. P. Butch, P. Syers, S. Ziemak, R. L. Greene, and J. Paglione, Hybridization, Inter-Ion Correlation, and Surface States in the Kondo Insulator $\mathrm{SmB}_{6}$, Phys. Rev. X 3, 011011 (2013). 